\title{
Association between Remnant-Like Particle Cholesterol and Gamma-Glutamyltransferase in Dyslipidemic Patients
}

\author{
Kazuhiko Kotani ${ }^{\mathrm{a}, \mathrm{b}}$ Naoki Sakane ${ }^{\mathrm{a}}$ \\ ${ }^{a}$ Division of Preventive Medicine, Clinical Research Institute, National Hospital Organization Kyoto Medical Center,

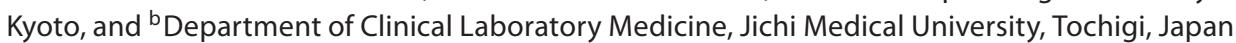

\section{Key Words}

Remnant lipoprotein • Remnant-like particle cholesterol • Gamma-glutamyltransferase $\cdot$ Atherosclerosis

\begin{abstract}
Objective: To investigate the correlation between gammaglutamyltransferase (GGT) and remnant-like particle cholesterol (RLP-C) in dyslipidemic patients. Subjects and Methods: General cardiometabolic variables, plus serum GGT and RLP-C levels, were measured in 118 dyslipidemic patients (59 males, mean age $58 \pm 13$ years). Correlation analyses of GGT with other variables, including RLP-C, were performed. $R \boldsymbol{e}$ sults: The median levels (interquartile ranges) of RLP-C and GGT were $0.21 \mathrm{mmol} / \mathrm{l}(0.14-0.35)$ and $26 \mathrm{IU} / \mathrm{l}$ (17-39), respectively. A stepwise multiple regression analysis revealed an independent, significant and positive correlation between GGT and RLP-C $(\beta=0.351, p<0.001)$, followed by male gender and BMI. Conclusion: There was a significant positive association between GGT and RLP-C, thereby indicating that the association between GGT and cardiovascular disease may be partly due to the interaction with RLP-C in dyslipidemic patients. Further research is therefore warranted to confirm the observed relationship.
\end{abstract}

Copyright $\odot 2012$ S. Karger AG, Basel

\begin{tabular}{|c|c|}
\hline KARGER & $\begin{array}{l}\text { (c) } 2012 \text { S. Karger AG, Basel } \\
1011-7571 / 13 / 0222-0189 \$ 38.00 / 0\end{array}$ \\
\hline $\begin{array}{l}\text { E-Mail karger@karger.ch } \\
\text { www.karger.com/mpp }\end{array}$ & $\begin{array}{l}\text { This is an Open Access article licensed under the terms of } \\
\text { the Creative Commons Attribution- NonCommercial-No- } \\
\text { Derivs } 3.0 \text { License (www.karger.com/OA-license), applica- } \\
\text { ble to the online version of the article only. Distribution for } \\
\text { non-commercial purposes only. }\end{array}$ \\
\hline
\end{tabular}

\section{Introduction}

Gamma-glutamyltransferase (GGT) is commonly used as an enzyme marker of liver dysfunction (i.e. fatty liver) [1]. The GGT is also known as an emerging risk marker for cardiovascular disease (CVD), regardless of gender [2-4]. Although it remains unclear whether GGT is an innocent bystander or a causal contributor to CVD $[2,3]$, it is known that GGT participates in glutathione metabolism with the extracellular degradation of the antioxidant glutathione, GSH, possibly exerting an oxidative stress-related function $[2,3]$. In addition, GGT is associated with lipid abnormalities [4]; thus, the predictive value of GGT for CVD may be assumed to be linked to lipid metabolism.

Remnant-like particle cholesterol (RLP-C) has received a lot of attention as a residual CVD risk marker besides the traditional CVD risk factors $[5,6]$. Remnantlike particles can be taken up by macrophages (forming foam cells), stimulate endothelial cells to express monocyte-specific chemotactic factor, and increase monocyte adhesion to the endothelium, thereby leading to atherosclerosis [5]. RLP-C reflects in vivo remnant lipoprotein/ lipid metabolism (remnant particles of chylomicron and very-low-density lipoprotein in particular) in relation to the liver function [7]. Although one would expect some

Kazuhiko Kotani, MD, PhD

Department of Preventive Medicine, Clinical Research Institute National Hospital Organization Kyoto Medical Center

1-1 Fukakusa mukaihata, Fushimi-ku, Kyoto 612-8555 (Japan)

Tel. +81 285587 386, E-Mail kazukotani@jichi.ac.jp 
association between RLP-C and GGT given their involvement in the liver pathophysiology, their associations have scarcely been examined. The aim of the present study was to investigate the association between GGT and RLP-C in dyslipidemic patients.

\section{Subjects and Methods}

A total of 118 dyslipidemic patients (59 male and 59 female, mean age $58 \pm 13$ years (range $30-80$ )) were enrolled in this study among patients presenting at our clinic and in the health care setting. Dyslipidemia was diagnosed according to the guidelines of the Japan Atherosclerosis Society (circulating concentrations of LDL cholesterol (LDL-C) $\geq 3.64 \mathrm{mmol} / \mathrm{l}$, triglycerides (TG) $\geq 1.69 \mathrm{mmol} / \mathrm{l}$, high-density lipoprotein cholesterol (HDL-C) $<1.04 \mathrm{mmol} / \mathrm{l}$ ). The inclusion criteria were nonsmokers, non-alcohol drinkers, and patients not taking current medications. The exclusion criteria were individuals who were pregnant, had acute infections or had a past history of cardio-/cerebrovascular, thyroid, collagen, kidney or viral liver diseases. The study was approved by the Kyoto Medical Center Ethics Committee, and all subjects gave their informed consent for participation.

The BMI was calculated as the weight divided by the height squared. The systolic blood pressure (SBP) and diastolic blood pressure (DBP) were measured in the right arm in a seated position using a mercuric sphygmomanometer with appropriate size cuffs. After an overnight fast, serum lipid panels, such as LDL-C, TG and HDL-C, plasma glucose and alanine aminotransferase (ALT) levels were measured with enzymatic methods. GGT was measured with an enzyme kinetic assay using L- $\gamma$-glutamyl-3carboxy-4-nitroanilide as a substrate [8]. RLP-C was measured with a homogeneous assay, where the cholesterol levels in remnants were assayed with the use of polyoxyethylene-polyoxybutylene block copolymer and phospholipase-D in the presence of cholesterol esterase and cholesterol oxidase [6].

The data are expressed as the means \pm SDs for parametric variables or the medians (interquartile ranges) for nonparametric variables. The parametric or nonparametric variables were determined based on Shapiro-Wilk and Kolmogorov-Smirnov analyses as well as the distribution figures of variable values. Differences between the groups were compared using unpaired t test. Pearson's correlation test (coefficient $r$ ) for the univariate relationship and a (stepwise) multiple linear regression analysis adjusted for all measured variables were utilized to examine the correlation between GGT and the measured variables, including RLP-C. The values of TG, RLP-C, ALT and GGT (as nonparametric variables) were log-transformed for these correlation analyses because of their skewed distributions. Spearman's rank correlation test (coefficient $\rho$ ) was also utilized for a univariate analysis of the relationship for nonparametric variables. In addition, a subgroup analysis by glucose states (hyperglycemic state defined as $\geq 7.0 \mathrm{mmol} / \mathrm{l}$ of fasting plasma glucose level $[9,10]$ ) was similarly performed in 26 patients (males 6 , females 20) with a mean age of $61 \pm 11$ years. This was compared to 92 patients (males 53, females 39) with a mean age of $57 \pm 13$ years who were not in a hyperglycemic state. $\mathrm{p}<0.05$ was considered statistically significant. The analyses were carried out using the SPSS ${ }^{\circledR}$ statistical package, version 16.0 (SPSS Inc., Chicago, Ill., USA).
Table 1. Correlation of each variable with GGT

\begin{tabular}{lcc}
\hline & $\mathrm{r}(\mathrm{p}$ value $)$ & $\beta$ (p value) \\
\hline Age, years & $-0.133(0.151)$ & not extracted \\
Gender, male & $0.439(<0.001)$ & $0.268(0.001)$ \\
$\mathrm{BMI}, \mathrm{kg} / \mathrm{m}^{2}$ & $0.389(<0.001)$ & $0.223(0.005)$ \\
$\mathrm{SBP}, \mathrm{mm} \mathrm{Hg}$ & $0.039(0.672)$ & not extracted \\
$\mathrm{DBP}, \mathrm{mm} \mathrm{Hg}$ & $0.201(0.030)$ & not extracted \\
LDL cholesterol, mmol/l & $0.034(0.719)$ & not extracted \\
Triglycerides, $\mathrm{mmol} / \mathrm{l}$ & $0.487(<0.001)$ & not extracted \\
HDL cholesterol, mmol/l & $-0.317(<0.001)$ & not extracted \\
Plasma glucose, $\mathrm{mmol} / \mathrm{l}$ & $0.171(0.063)$ & not extracted \\
RLP cholesterol, mmol/l & $0.515(<0.001)$ & $0.351(<0.001)$ \\
\hline
\end{tabular}

The data are expressed as $r$ (Pearson's correlation coefficient) and $\beta$ (regression coefficient by a stepwise multiple regression analysis adjusted for all listed variables). The GGT, triglycerides and RLP cholesterol values were analyzed after log-transformation because of their skewed distributions. Significance level: $\mathrm{p}<$ 0.05 .

\section{Results}

The mean/median levels of the measured variables were as follows: BMI $24.1 \pm 3.5 \mathrm{~kg} / \mathrm{m}^{2}$; SBP $136 \pm 21 \mathrm{~mm}$ $\mathrm{Hg}$; DBP $79 \pm 11 \mathrm{~mm} \mathrm{Hg}$; LDL-C $4.17 \pm 0.79 \mathrm{mmol} / \mathrm{l}$; TG $1.44(1.03-2.47) \mathrm{mmol} / \mathrm{l}$; HDL-C $1.62 \pm 0.49 \mathrm{mmol} / \mathrm{l}$; RLP-C $0.21(0.14-0.35) \mathrm{mmol} / \mathrm{l}$, and GGT 26 (17-39) IU/l.

The correlations between the GGT level and other variables are listed in table 1. Pearson's correlation test showed that the GGT level was significantly and positively correlated with male gender, BMI, DBP, TG and RLP-C, while it was significantly and inversely correlated with HDL-C. Spearman's rank correlation test also showed the GGT level to correlate significantly and positively with TG $(\rho=0.486, \mathrm{p}<0.001)$ and RLP-C $(\rho=$ $0.500, \mathrm{p}<0.001)$.

A subsequent stepwise multiple linear regression analysis for GGT revealed that there was an independent, significant and positive correlation between GGT and RLP$\mathrm{C}$, followed by male gender and BMI (table 1). There was an independent, significant and positive correlation between GGT and RLP-C $(\beta=0.385, p<0.001)$, followed by male gender $(\beta=0.309, p=0.001)$ and $\mathrm{BMI}(\beta=0.233$, $\mathrm{p}=0.008)$, when the multiple linear regression analysis for GGT was performed in a forced entry method of adjustment with all measured variables of age, gender, BMI, SBP, LDL-C, HDL-C, glucose and RLP-C (SBP and RLP$\mathrm{C}$ only were entered into this model because of a close colinearity with DBP (for SBP, $r=0.750, p<0.001$ ) and 
Table 2. Correlation of each variable with GGT according to the fasting plasma glucose levels

\begin{tabular}{|c|c|c|c|c|}
\hline & \multicolumn{2}{|c|}{ Glucose $<7.0 \mathrm{mmol} / \mathrm{l}(\mathrm{n}=92)$} & \multicolumn{2}{|c|}{ Glucose $\geq 7.0 \mathrm{mmol} / \mathrm{l}(\mathrm{n}=26)$} \\
\hline & $\mathrm{r}$ ( $\mathrm{p}$ value $)$ & $\beta$ (p value) & $\mathrm{r}$ ( $\mathrm{p}$ value $)$ & $\beta$ (p value) \\
\hline Age, years & $-0.116(0.272)$ & not extracted & $-0.300(0.136)$ & not extracted \\
\hline Gender, male & $0.446(<0.001)$ & $0.332(<0.001)$ & $0.291(0.150)$ & not extracted \\
\hline BMI, kg/m² & $0.332(0.001)$ & $0.210(0.021)$ & $0.470(0.015)$ & $0.220(0.176)$ \\
\hline $\mathrm{SBP}, \mathrm{mm} \mathrm{Hg}$ & $0.007(0.949)$ & not extracted & $-0.111(0.590)$ & not extracted \\
\hline $\mathrm{DBP}, \mathrm{mm} \mathrm{Hg}$ & $0.130(0.216)$ & not extracted & $0.268(0.185)$ & not extracted \\
\hline LDL cholesterol, mmol/l & $-0.393(<0.001)$ & not extracted & $0.146(0.477)$ & not extracted \\
\hline Triglycerides, mmol/l & $0.393(<0.001)$ & not extracted & $0.686(<0.001)$ & not extracted \\
\hline HDL cholesterol, mmol/l & $0.400(<0.001)$ & not extracted & $-0.219(0.282)$ & not extracted \\
\hline Plasma glucose, mmol/l & $-0.009(0.935)$ & not extracted & $0.138(0.500)$ & not extracted \\
\hline RLP cholesterol, mmol/l & $0.427(<0.001)$ & $0.289(0.002)$ & $0.696(<0.001)$ & $0.605(0.001)$ \\
\hline
\end{tabular}

The data are expressed as $r$ (Pearson's correlation coefficient) and $\beta$ (regression coefficient by a stepwise multiple regression analysis adjusted for all listed variables). The GGT, triglycerides and RLP cholesterol values were analyzed after log-transformation because of their skewed distributions. Significance level: $\mathrm{p}<0.05$.

TG (for RLP-C, $\mathrm{r}=0.802, \mathrm{p}<0.001 ; \rho=0.800, \mathrm{p}<0.001$ ), respectively).

In addition, the subanalysis by glucose states showed that the patients in a hyperglycemic state $(0.30(0.21-$ $0.65) \mathrm{mmol} / \mathrm{l}$ ) exhibited a significantly higher RLP-C level than those that were not in a hyperglycemic state $(0.18(0.13-0.32) \mathrm{mmol} / \mathrm{l}, \mathrm{p}=0.018)$. The patients in a hyperglycemic state tended to show a higher GGT level (24 (16-37) IU/l) relative to those that were not in a hyperglycemic state (34 (21-40) IU/l, p = 0.061). The correlations between the GGT level and other variables by glucose states are listed in table 2. Pearson's correlation test showed that the GGT level was significantly and positively correlated with male gender, BMI, TG, HDL-C and RLP-C, and it was significantly and inversely correlated with LDL-C in the patients that were not in a the hyperglycemic state, while the GGT level was significantly and positively correlated with BMI, TG and RLP-C in the patients in a hyperglycemic state. Spearman's rank correlation test also showed that the GGT level was significantly and positively correlated with TG $(\rho=0.399, \mathrm{p}<0.001$ in the patients who were not in a hyperglycemic state; $\rho=0.582, p=0.002$ in the patients in a hyperglycemic state $)$ and RLP-C $(\rho=0.402, \mathrm{p}<0.001$ in the patients who were not in a hyperglycemic state; $\rho=0.631, p=0.001$ in the patients in a hyperglycemic state). A subsequent stepwise multiple linear regression analysis revealed an independent, significant and positive correlation between GGT and RLP-C, male gender or BMI in the patients that were not in a hyperglycemic state, while there was an in- dependent, significant and positive correlation between GGT and RLP-C in the patients in a hyperglycemic state (table 2). When a multiple linear regression analysis was performed in a forced entry method of adjustment with all measured variables as aforementioned similarly, there was an independent, significant and positive correlation between GGT and RLP-C ( $\beta=0.310, p=0.003)$, male gender $(\beta=0.349, \mathrm{p}=0.001)$ or $\mathrm{BMI}(\beta=0.240, \mathrm{p}=0.018)$ in the patients who were not in a hyperglycemic state, while an independent, significant and positive correlation was seen between GGT and RLP-C $(\beta=0.700, p=$ $0.003)$ in the patients in a hyperglycemic state.

Furthermore, the median level of ALT was 19 (14-26) IU/l in all of the patients. The GGT level significantly and positively correlated with ALT $(r=0.550, p<0.001 ; \rho=$ $0.551, \mathrm{p}<0.001)$. The ALT level was significantly and positively correlated with RLP-C $(r=0.345, \mathrm{p}<0.001 ; \rho=$ $0.500, p<0.001)$. Using the ALT level, instead of GGT, as a dependent variable, a multiple linear regression analysis (as aforementioned) showed no significant correlation between ALT and RLP-C according to a stepwise model (not extracted) or a forced entry model of adjustment with all variables $(\beta=0.140, p=0.164)$.

\section{Discussion}

The present study demonstrated that GGT was independently, significantly and positively associated with RLP-C in dyslipidemic patients. The significant correla- 
tion of GGT with male gender and BMI was expected based on previous studies $[2,4]$. The significant correlation between GGT and RLP-C suggests that the link between the GGT level and the development of CVD may be, in part, due to its association with RLP-C in these patients. This finding is considered to be important and valuable since the roles of GGT on the incidence of CVD have not yet been completely elucidated $[2,3]$.

The precise mechanisms responsible for the relationship between the GGT level and RLP-C are unclear, but GGT is presumably correlated with RLP-C. Hepatic dysfunction leads to hepatic overproduction of remnant particles [7]. In fact, hypertriglyceridemia and existing triglyceride-rich lipoproteins, partly linked with the metabolism of remnants, can be significantly related to elevated GGT or fatty liver [11], although it has been debated whether (nonalcoholic) fatty liver is closely associated with GGT $[4,11,12]$. Currently, the liver is recognized as one of the organs that mediates oxidative stress and lipoprotein/lipid metabolism [13]. The GGT and ALT levels showed nearly normal ranges in the present study patients, so even though there was hepatic dysfunction, the degree was possibly not very severe. Therefore, this indicates that GGT may not have a threshold to increase RLPC. In addition, the present study showed that there was a clear correlation between GGT and RLP-C relative to the correlation between ALT and RLP-C. Both GGT and ALT can reflect hepatic dysfunction, but it is suggested that GGT has a greater influence on atherosclerosis, relative to AST [14]. Simultaneous consideration of GGT and RLP-C may be useful to evaluate the pathophysiological processes of oxidative stress and lipoprotein metabolism in the liver in association with the development of CVD.
Remnant particles are reportedly elevated in diabetic patients [15]. Interestingly, the patients in a hyperglycemic state seemed to show a greater correlation between GGT and RLP-C in comparison to the patients that were not in a hyperglycemic state in the subanalysis by glucose states in the present study. This might be partially due to a high level of GGT and RLP-C in the patients in a hyperglycemic state relative to those that were not in a hyperglycemic state. Any association between this degree of correlation and the predisposition of the occurrence of CVD among hyperglycemic patients merits further investigation.

This study had the limitations associated with a small sample size and a cross-sectional design. This design cannot completely establish causation. The choice of a specific diseased population due to strict criteria (i.e. nonalcohol drinkers, dyslipidemic and not-medicated patients) can produce a selection bias, although the recruitment of patients may change the influence of various confounding factors related to GTP and RLP-C (these variables are easily influenced by lifestyle-related factors). No data on CVD-related outcomes were available. Therefore, caution must be exercised in interpreting the current results.

\section{Conclusion}

GGT was independently, significantly and positively correlated with RLP-C in dyslipidemic patients. This finding suggests that the GGT-CVD relationship may be at least partly due to the link between GGT and RLP-C, although further research is needed.

\section{References}

1 Lim JS, Lee DH, Park JY, Jin SH, Jacobs DR Jr: A strong interaction between serum gammaglutamyltransferase and obesity on the risk of prevalent type 2 diabetes: results from the Third National Health and Nutrition Examination Survey. Clin Chem 2007;53:1092-1098.

2 Kazemi-Shirazi L, Endler G, Winkler S, Schickbauer T, Wagner O, Marsik C: Gamma-glutamyltransferase and long-term survival: is it just the liver? Clin Chem 2007;53: 940-946.

-3 Turgut O, Tandogan I: Gamma-glutamyltransferase to determine cardiovascular risk: shifting the paradigm forward. J Atheroscler Thromb 2011;18:177-181.
4 Kim DJ, Noh JH, Cho NH, Lee BW, Choi YH, Jung JH, Min YK, Lee MS, Lee MK, Kim KW: Serum gamma-glutamyltransferase within its normal concentration range is related to the presence of diabetes and cardiovascular risk factors. Diabet Med 2005;22:1134-1140.

5 Nakajima K, Nakano T, Tanaka A: The oxidative modification hypothesis of atherosclerosis: the comparison of atherogenic effects on oxidized LDL and remnant lipoproteins in plasma. Clin Chim Acta 2006;367: $36-47$.

6 Kotani K, Sakane N, Taniguchi N: Effect of ezetimibe on remnant-like particle cholesterol in subjects with metabolic syndrome. Med Princ Pract 2012;21:134-138.
7 Adiels M, Olofsson SO, Taskinen MR, Borén $\mathrm{J}$ : Overproduction of very low-density lipoproteins is the hallmark of the dyslipidemia in the metabolic syndrome. Arterioscler Thromb Vasc Biol 2008;28:1225-1236.

$>8$ Persijn JP, van der Slik W: A new method for the determination of gamma-glutamyltransferase in serum. J Clin Chem Clin Biochem 1976;14:421-427.

9 World Health Organization: Definition and Diagnosis of Diabetes Mellitus and Intermediate Hyperglycemia. Report of a WHO/IDF Consultation. Geneva, World Health Organization, 2006. 
10 American Diabetes Association: Diagnosis and classification of diabetes mellitus. Diabetes Care 2012;35:S64-S71.

- 11 Sakugawa H, Nakayoshi T, Kobashigawa K, Nakasone H, Kawakami Y, Yamashiro T, Maeshiro T, Tomimori K, Miyagi S, Kinjo F, Saito A: Metabolic syndrome is directly associated with gamma glutamyl transpeptidase elevation in Japanese women. World J Gastroenterol 2004;10:1052-1055.
12 Marchesini G, Avagnina S, Barantani EG, Ciccarone AM, Corica F, Dall'Aglio E, Dalle Grave R, Morpurgo PS, Tomasi F, Vitacolonna E: Aminotransferase and gamma-glutamyltranspeptidase levels in obesity are associated with insulin resistance and the metabolic syndrome. J Endocrinol Invest 2005; 28:333-339.

13 de Ferranti S, Mozaffarian D: The perfect storm: obesity, adipocyte dysfunction, and metabolic consequences. Clin Chem 2008; 54:945-955.
14 Mikhailidis DP, Lioudaki E, Ganotakis ES: Liver enzymes: potential cardiovascular risk markers? Curr Pharm Des 2011;17:36323643.

15 Schaefer EJ, McNamara JR, Shah PK, Nakajima K, Cupples LA, Ordovas JM, Wilson PW, Framingham Offspring Study: Elevated remnant-like particle cholesterol and triglyceride levels in diabetic men and women in the Framingham Offspring Study. Diabetes Care 2002;25:989-994. 